\title{
Metastatic Malignant Neoplasm in the
} Pleura

National Cancer Institute

\section{Source}

National Cancer Institute. Metastatic Malignant Neoplasm in the Pleura. NCI Thesaurus.

Code C3579.

The spread of a malignant neoplasm to the pleura from an adjacent or distant anatomic site. 